\title{
White light generation from CdS nanoparticles illuminated by UV-LED
}

\author{
A.M. Suhail ${ }^{1}$, M.J. Khalifa ${ }^{1,2, a}$, N.M. Saeed ${ }^{1}$, and O.A. Ibrahim ${ }^{1}$ \\ 1 Physics Department, College of Science, University of Baghdad, Iraq \\ 2 Physics Department, College of Science, Wassit University, Iraq
}

Received: 15 September 2009 / Accepted: 24 November 2009

Published online: 3 February 2010 - (c) EDP Sciences

\begin{abstract}
The generation of white light from CdS nanoparticles (NPs) illuminated by UV-LED is presented. The synthesis of CdS nanoparticles from the reaction of $\mathrm{CdCl}_{2}$ and the sulfur dissolved in oleylamine in 1:2 mole ratio was used in the preparation of CdS nanocrystalline. The PMMA film doped with CdS nanoparticles was prepared. The generation of white light was found from illumination of the CdS/polymer composite film by UV-LED of power $5 \mathrm{~mW}$ at $\lambda=385 \mathrm{~nm}$. The white light generation mechanism was explaining depending on mixing colors from the illuminated CdS nanoparticles. The surface levels formed in the CdS optical energy band due to nanostructure was estimated from Poisson equation designed for this state. The chromaticity coordinates of the generated white light was measured by the help of photoluminescence (PL) spectrum and the correlated color temperature was found to be about $4000 \mathrm{~K}$.
\end{abstract}

\section{Introduction}

Since $25 \%$ of the global electricity consumption is used for lighting, thus the white light generation have attracted great interest for saving the electrical energy consumption in order to reduce the operating expense [1]. The solid state lighting (SSL) by using the tradition phosphor is not new, and different schemes have been reported to generate white light [2-8]. One of the most popular ways is the blend red, green and blue phosphor in appropriate ratio to generate the white light $[3,7]$. This important of the SSL not only due to its financial benefits, but become the SSL is predicted to reduce the carbon emission highly which lead to clean environment.

The energy efficiency of SSL in converting electricity into light is about $50 \%$ whereas for traditional lamp it's around $1 \%$. In addition, SSL offer ultra-long lifetimes for the lamps and the source become, ragged and compact.

The white nanoluminophors have attracted more attention for their potential using solid-state lighting. Usually the materials used in white nanoluminophors are semiconductors like CdS, CdSe, $\mathrm{ZnS}$ and ZnSe with size in order of $1-10 \mathrm{~nm}$ [6].

Since the nanostructure have a large number of surface atoms compare with the bulk, thus the characteristics of the surface atoms have more effect on the main physical and chemical properties of the material.

The surface states appear as trap-states in the main energy gap of the nanosemiconductor; therefore when it's illuminated by UV light the states emit radiation

\footnotetext{
a e-mail: ma_11prince@yahoo.com
}

with relatively high quantum efficiency [9]. The traprich nanocrystals are much useful than the emission from doped-semiconductor nanocrystals. It has been notice that the Mn-doped nanocrystal emits the white light as results of their collective emission in blue from CdS and in yellow from $\mathrm{Mn} d$ - $d$ level. However, the quantum efficiency of the emitted white light is very low $[9,10]$.

In this work the CdS NCs quantum dots are prepared from chemical synthesis. The trap-rich CdS NCs illuminated by GaN UV-LED to generate the white light with good efficiency.

\section{Experimental work}

\subsection{Preparation of colloidal CdS nanoparticle}

CdS nanoparticles were prepared as a colloidal from reaction of cadmium chloride and oleylamine (Cd-oleylamine complex) with sulfite oleylamine (S-oleylamine).

The S-oleylamine of concentration $0.15 \mathrm{M}$ was prepared by adding 0.75 mmole of sulfur to $5 \mathrm{ml}$ of oleylamine solution. The mixture was mixed by magnetic stirrer until a complete dissolution, and the color of solution was change from yellow to red, this mean that the S-oleylamine was formed.

Cd-oleylamine complex of concentration (0.15 M) was prepared by adding 1.5 mmole of cadmium chloride to $10 \mathrm{ml}$ of oleylamine solution (molecular structure $\mathrm{C}_{18} \mathrm{H}_{37} \mathrm{~N}$ supplied by Fluka). The mixture was heated to 
$90{ }^{\circ} \mathrm{C}$ with stirring using magnetic stirrer. After the complete dissolution of cadmium chloride in oleylamine solution, the mixture was left on magnetic stirrer for $30 \mathrm{~min}$ at the same temperature, to form Cd-oleylamine complex.

The Cd-oleylamine complexes were heated at $170{ }^{\circ} \mathrm{C}$ for 20 min under argon flow, S-oleylamine solution was injected under gentle stirring into the hot reaction mixture. CdS nanoparticle was prepared by reaction of S-oleylamine solution with Cd-oleylamine complexes with mole ratio $2: 1$ [11].

The hot reaction mixture was held at $170{ }^{\circ} \mathrm{C}$ with stirred for $6 \mathrm{~h}$, and then the mixture cooled and added to large volume of ethanol at room temperature to quench the reaction and to precipitated. The yellow precipitate of CdS nanoparticle formed.

The yellow precipitate of CdS nanoparticle washed several times by ethanol for removing the excess of oleylamine, separated by centrifugation and dried under room temperature and atmosphere pressure to make it a powder.

\subsection{Preparation of CdS/PMMA composite films}

After the preparation of CdS nanoparticle, CdS/PMMA composite film was prepared by dissolving $1 \mathrm{gm}$ of PMMA in $10 \mathrm{ml}$ of chloroform and stirred with help of magnetic stirrer and heating to $70{ }^{\circ} \mathrm{C}$ for $10 \mathrm{~min}$. A CdS nanopowder of $0.02 \mathrm{gm}$ weight was dispersed in PMMA solution and left it on the same conditions for $15 \mathrm{~min}$, then poured into flat-bottomed petri dish to form film with concentration of $13.8 \mathrm{mmol} / \mathrm{ml}$ and thickness of $\sim 350 \mu \mathrm{m}$.

\section{Test of the prepared samples}

\subsection{X-ray diffraction}

The X-ray diffraction (XRD) pattern of CdS nanoparticle powder was recorded by SHIMADZU XRD-6000. The $\mathrm{X}$-ray diffractometer use $\left(\mathrm{CuK}_{\alpha}\right.$ radiation line of wavelength of $1.54 \AA$ in $2 \theta$ range from $20^{\circ}$ to $60^{\circ}$.

\subsection{Absorption and PL spectra}

UV-Vis absorption spectrum of the samples was recorded by OPTIMA SP_3000 UV_Vis spectrophotometer covering a range from (200-1100) $\mathrm{nm}$.

The photoluminescence spectrum (PL) was taken by SL 174 spectrofluorometer covering a range from (300-900) nm. The A atomic force microscopy (AFM) was used to study the morphology of the prepared CdS nanoparticles and to determine the particles dimensions range and their statistical distribution.

The illumination of the CdS/PMMA composite films was carried on by a GaN UV-LED of a power of $5 \mathrm{~mW}$ in order to generate the white light.

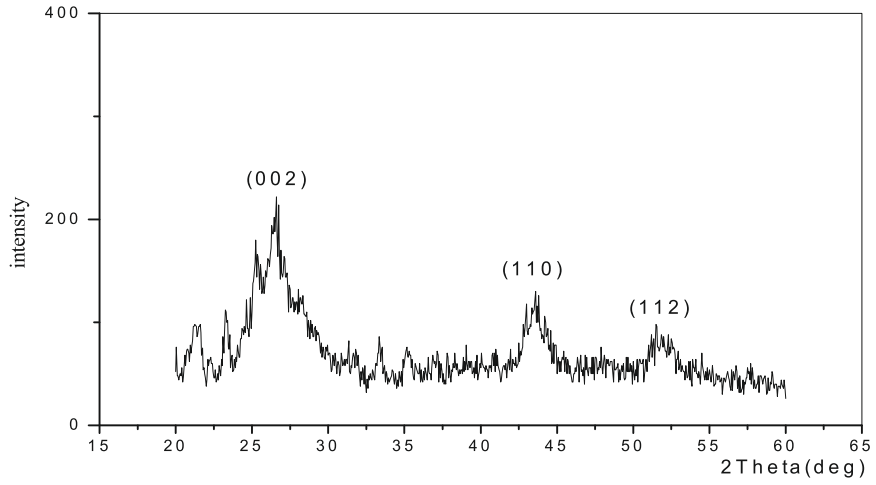

Fig. 1. The XRD pattern of CdS nanoparticle.

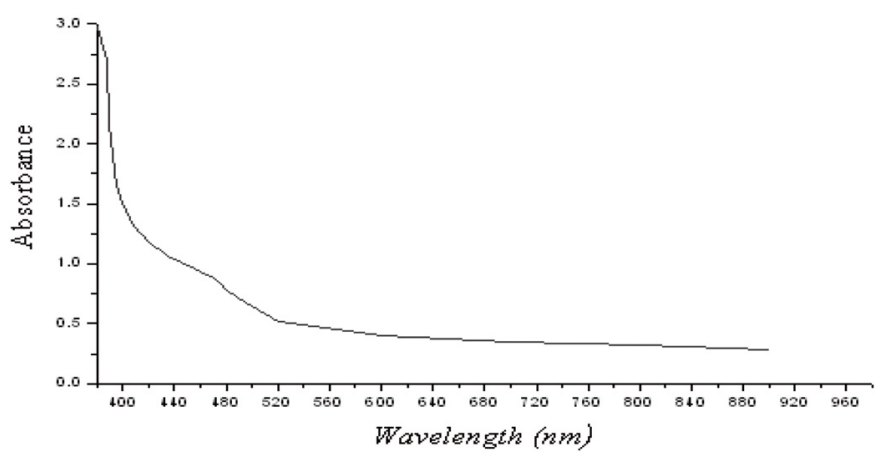

Fig. 2. UV-Vis absorption spectrum of CdS nanoparticle.

\section{Result and discussion}

The XRD of the yield CdS nanoparticles was tested by $\mathrm{k}_{\alpha} \mathrm{Cu}$ source line of $1.54 \AA$ wavelength as mentioned before. The XRD pattern was shown in Figure 1. The figure shows broad peaks which give evidence of the formation of the nanostructure. Using the width of (002) peak which appears at angle $26.5^{\circ}$ on $2 \theta$ scale in Scherrer relation [12]:

$$
D=\frac{0.9 \lambda}{\beta \cos \theta},
$$

where $D$ is the grain size, $\lambda$ is the wavelength, $\beta$ represents the full width at half maximum $(F W H M)$ in degree and $\theta$ is the diffraction angle, the size of the formed nanoparticles was found to be about $5 \mathrm{~nm}$.

The UV-Vis absorption spectrum of the colloidal CdS nanoparticle is shown in Figure 2. The spectrum shows an absorption peak at $350 \mathrm{~nm}$ and absorption edge at $480 \mathrm{~nm}$ which indicated that there is a blue shift of about $32 \mathrm{~nm}$ due to the quantum confinement effect.

The AFM image of the surface morphology of CdS film gives a good indicator for formation of the CdS nanoparticles. The particle size which determined from AFM is about $8 \mathrm{~nm}$, as shown in Figure 5 .

The photoluminescence emission spectrum (PL) of CdS nanoparticle solution excited by $350 \mathrm{~nm}$ line is shown in Figure 3. The spectrum shows a peak at $485 \mathrm{~nm}$ which can be referred to the direct band transition and two peaks at 580 and $630 \mathrm{~nm}$ which where attributed to the formation of surface states in the energy gap. This observation 


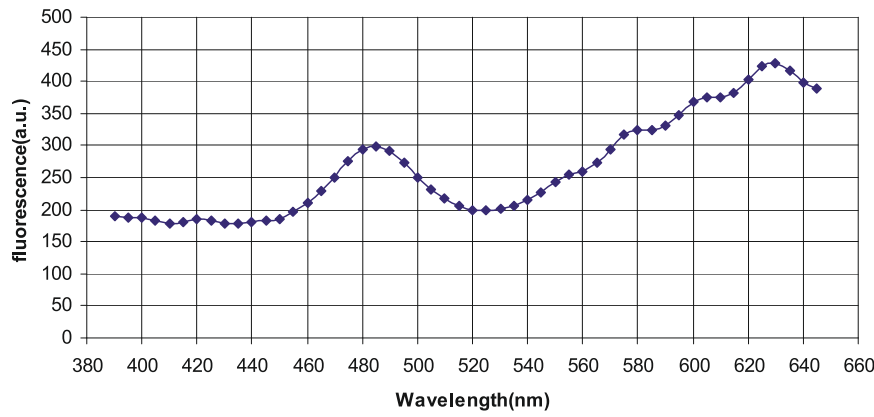

Fig. 3. (Color online) Photoluminescence emission spectrum of CdS nanoparticle.

in the doped CdS nanoparticles was mentioned by other workers [10].

The surface trapping peaks are attributed to the formation of the surface states in the energy gap due to nanostructure of the prepared CdS. In order to check the emission peaks observed in the PL spectrum a mathematical model describing the spatial variation of the potential at the grain boundaries due to the trapping of charge carriers was proposed. The model based on solution of Poisson equation at the grain boundary, as the following [13]:

$$
\nabla^{2} V=-\frac{\rho}{\epsilon}
$$

where $(\rho)$ is the charge density in the sample. The charge distribution among the boundary is considered as its distribution in the junction. Usually the charge concentration changes in state-like manner but actually in diffused junction the concentration varies gradually. The practical distribution of the charge in the junction and in the grain boundaries can be written as [14]:

$$
\rho=\rho_{\circ} \sec h\left(\frac{x}{a}\right) \tanh \left(\frac{x}{a}\right),
$$

where $\rho_{\circ}=N e \cong 10^{3} \mathrm{c} / \mathrm{m}^{3} ; N$ is the carriers concentration, $e$ is the electronic charge, and $a$ is the grain size.

Solving equation (2) using the boundary conditions as follow:

- the charge concentrations $\rho=0$ at $x=0 ; \rho=\rho_{\circ}$ at $x=a$; the electric field $E=0$ at $x \gg a$ thus $\varepsilon$ which represents the surface state energy was found to be:

$$
\varepsilon=\frac{2 \rho_{\circ} a^{2}}{\in}\left(\tan ^{-1} e^{x / a}-\frac{\pi}{4}\right) e
$$

- using the values of $\rho_{\circ}=10^{3} \mathrm{c} / \mathrm{m}^{3}$, and the dielectric constant of the CdS samples $\epsilon_{r}=5.7$, the values of $\varepsilon$ for grain sizes $(a)$ which is in order of 4.6 and $8 \mathrm{~nm}$ were calculated.

The AFA image of the CdS nanoparticles show that the histogram of the percentage of CdS NCs as a function of the grain size as in Figure 6.

It can be noticed from the histogram that the major particles have a grain sizes in the range 6-12 nm. Thus

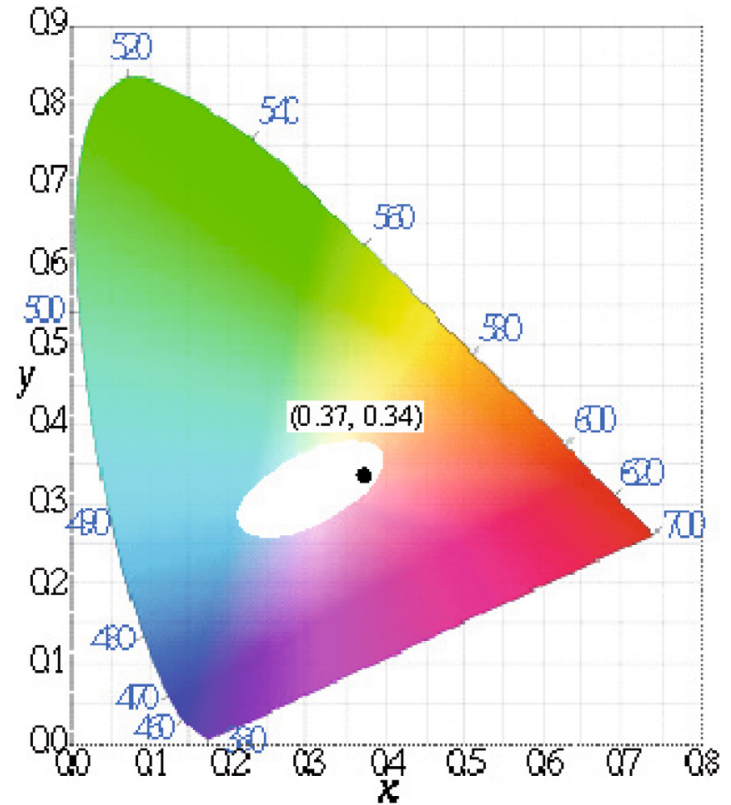

(a)

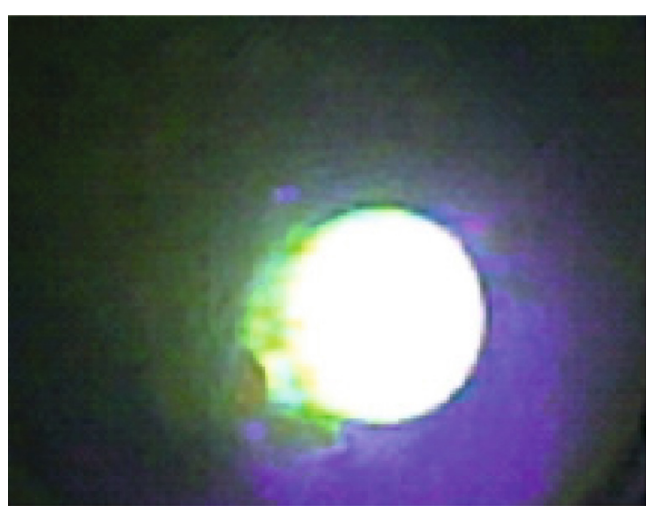

(b)

Fig. 4. (Color online) (a) Tristimulus coordinates of CdS nanoparticle $(0.37,0.34)$ on the CIE 1931 chromaticity diagram. (b) A photograph of white light generation from illuminated CdS/PMMA composite film by UVGaN-LED.

the values of $\varepsilon$ were calculated from equation (4) for the grain sizes mentioned above. The corresponding values of $\varepsilon$ were found by applying equation (4) as $0.1611,0.3625$ and $1.776 \mathrm{eV}$, respectively.

The appearance of the different peaks in the PL spectrum can be attributed to above range of the nanostructures dimension in the prepared CdS nanocrystal film.

The mid-gap trap states or the surface states of the above values were illustrated in Figure 7.

The emission due to transition from the CB to the mid-gap surface states were centered around 698,602 and $548 \mathrm{~nm}$ as show in Figure 7.

The CdS nanocrystal dispersed in PMMA matrix was illuminated by GaN UV-LED to activate the broad surface-state emission over the entire visible range to generate light close to white in color as shown in Figure 4a. 


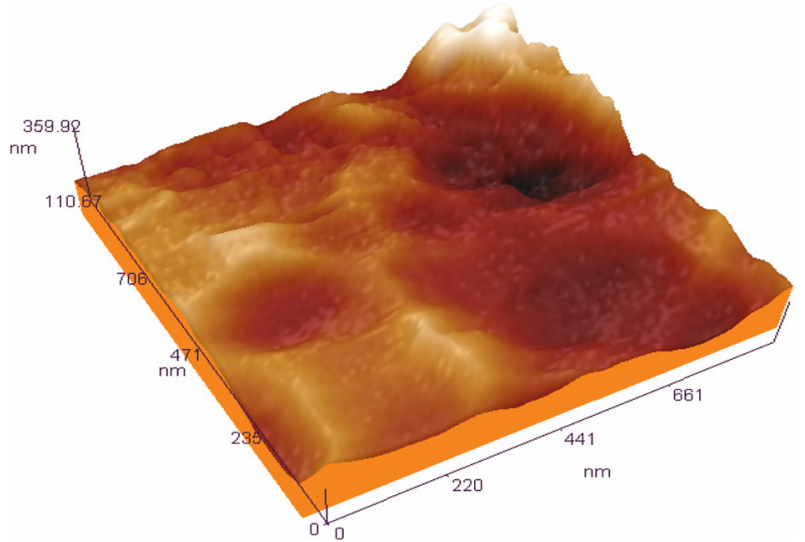

Fig. 5. (Color online) AFM image of CdS nanofilm prepared by chemical bath deposition.

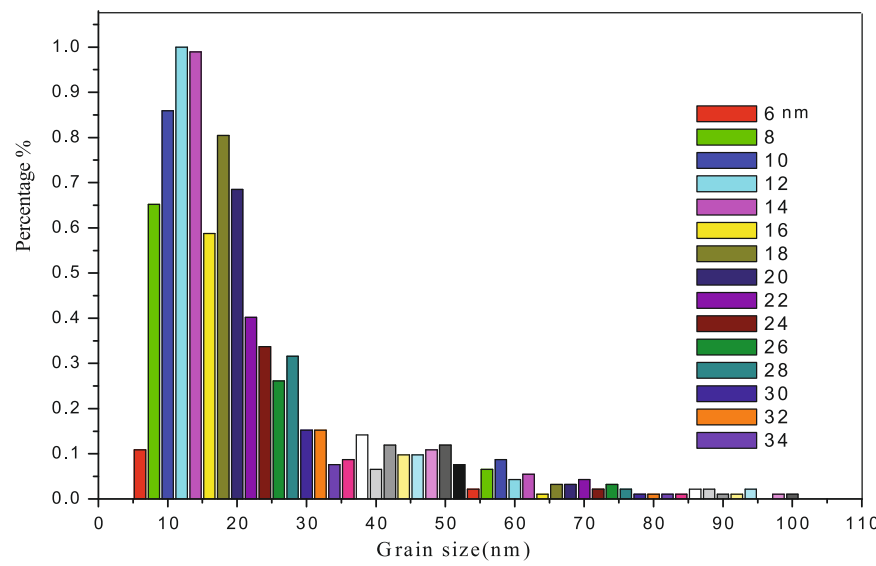

Fig. 6. (Color online) The percentage of the CdS NPs as a function of grain size.

\section{Chromaticity coordinates and correlated color temperature (CCT)}

The white-light produced by LEDs, or any other light sources for general lighting, should have an acceptable white color in order to show all the colors of illuminated objects appropriately. Since the color of light is expressed by the CIE colorimetry system, the spectrum of a given light is weighted by the $X Y Z$ color matching functions [15]. The resultant three weighted integral values of $X, Y$ and $Z$ are calculated from the PL spectrum by finding the area under the curve for the three peaks in red, green and blue centered around 485, 580 and $630 \mathrm{~nm}$.

The chromaticity coordinates $x, y$ on CIE colorimetry system are found from $X, Y$ and $Z$ as follow:

$$
\begin{aligned}
& x=X /(X+Y+Z)=0.37 \\
& y=Y /(X+Y+Z)=0.34
\end{aligned}
$$

$X, Y$, and $Z$, which are roughly the area under the curve of the emitted red, green and blue in PL spectrum, respectively.

Any color of light can be expressed by the chromaticity coordinate $(x, y)$ on the CIE $1931(x, y)$ chromaticity

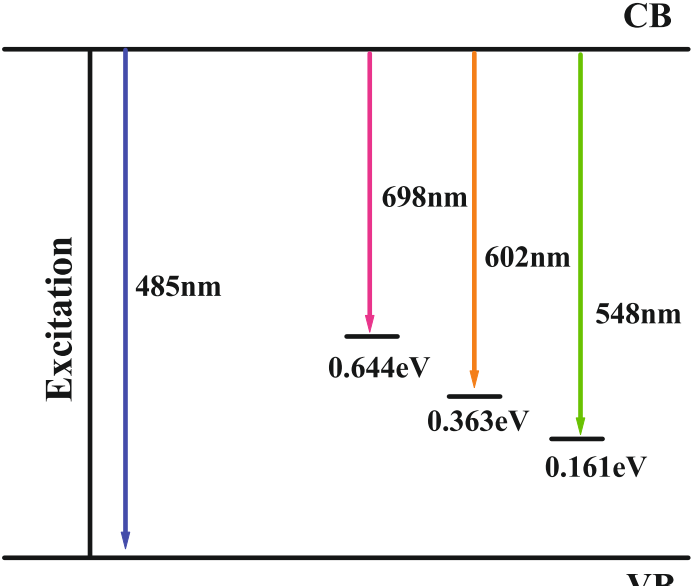

Fig. 7. (Color online) The mid-gap trap state from the CB-Trap state transition.

diagram. The white light which produced from our CdS nanoparticles has tristimulus coordinates $(x, y)=(0.37$, $0.34)$.

We can verify the correlated color temperature (CCT) for the resulting white light by using McCamy's approximation algorithm to estimate the CCT from the xy chromaticities $[15,16]$ :

$$
\mathrm{CCT}=-449 n^{3}+3525 n^{2}-6823.3 n+5520.33 \ldots,
$$

where

$$
n=\frac{x-0.3320}{y-0.1858}
$$

By using the values of $x$ and $y$ as determined before, the color temperature of the produced white light was found to be about $4000 \mathrm{~K}$.

The value of chromaticity coordinates were used in chromaticity diagram as shown in Figure 4 a to show that the output light of the emission lines registered in $\mathrm{PL}$ spectrum is in the white light region and this result was confirmed by photograph of the light emitted from the CdS/PMMA composite film illuminated by GaNUV-LED as shown in Figure $4 \mathrm{~b}$.

\section{Conclusion}

White light emission has been produced from illumination CdS NCs (with an average of $6 \mathrm{~nm}$ ) by GaNUV-LED. The peaks which appear in PL spectrum of CdS NCs result from direct band transition and surface-state transitions. CdS NCs illuminated by GaNUV-LED produce white light with chromaticity coordinates $(0.37,0.34)$, which is within the white region of the CIE 1931 diagram. These NCs exhibit a blue shift in its absorption spectrum, it is about $35 \mathrm{~nm}$. Results appeared that no change in the emission spectrum of samples either in solution or in the solid state. 
A.M. Suhail et al.: White light generation from CdS nanoparticles illuminated by UV-LED

\section{References}

1. J.Y. Tsao, Light Emitted diodes (LEDs) for general Illumination, OIDA technology report (2002)

2. A.H. Mueller, M.A. Petruska, M. Achermann, D.J. Werder, E.A. Akhadov, D.D. Koleske, M.A. Hoffbauer, V.I. Klimov, Nano Lett. 5, 1039 (2005)

3. H.S. Chen, S.J.J. Wang, C.J. Lo, J.Y. Chi, Appl. Phys. Lett. 86, 131905 (2005)

4. Y. Yang, M. Lowry, C.M. Schowalter, S.O. Alamode, J.O. Escobedo, X. Xiangyang, H. Zhang, T.J. Jensen, F.R. Fronczek, I.M. Warner, R.M. Strongin, J. Am. Chem. Soc. 128, $14081(2006)$

5. Y. Li, A. Rizzo, R. Kingsland, G. Gigli, Adv. Mater. 18, $2545(2006)$

6. M.J.II. Bowers, J.R. McBride, S.J. Rosenthal, J. Am. Chem. Soc. 127, 15378 (2005)
7. M. Ali, S. Chattopadhyay, A. Nag, A. Kumar, S. Sapra, S. Chakraborty, D.D. Sarma, Nanotechnology 18, 075401 (2007)

8. S. Sapra, S. Mayilo, T.A. Klar, A.L. Rogach, J. Feldmann, Adv. Mater. 19, 569 (2007)

9. S. Nizamoglu, G. Zengin, H.V. Demir, Appl. Phys. Lett. 92, $031102(2008)$

10. A. Nag, D.D. Sarma, J. Phys. Chem. C 111, 13641 (2007)

11. J. Joo, B. Na, T. Yu, J. Ho Yu, Y.W. Kim, F. Wu, J.Z. Zhang, T. Hyeon, J. Am. Chem. Soc. 125, 11100 (2003)

12. A.L. Patterson, Phys. Rev. 56, 978 (1939)

13. S.S. Islam, Semiconductor physics and Devices (Oxford University Press, Oxford, 2005), p. 228

14. W.H. Hayt, Engineering electromagnetics (McGraw-Hill, New York, 1989), pp. 201-203

15. CIE Free Document for download, http://www.cie.co. at/main/freepubs.html

16. C.S. McCamy, Color Res. Appl. 17, 142 (2007)

To access this journal online: www.edpsciences.org 